\title{
La calidad del cuidado prenatal como factor de intervención para reducir las complicaciones obstétricas y neonatales
}

\author{
Ricardo Figueroa-Damián* \\ Instituto Nacional de Perinatología, Ciudad de México, México
}

Con este número, a pesar de las limitaciones técnico-administrativas que hemos tenido durante este año, logramos mantener durante el año de 2019 la publicación de Perinatología y Reproducción Humana (RPRH). Esperamos que en el próximo año haya menos problemas y logremos la regularidad debida de esta publicación, que es el órgano oficial de difusión del Instituto Nacional de Perinatología.

Agradecemos a los colaboradores de la revista, quienes, a pesar de la intermitencia en su publicación durante este año, han continuado enviando trabajos para evaluar su publicación en RPRH, y han esperado con paciencia la reactivación y continuidad debida y necesaria de la revista.

Como es habitual en la RPRH, en este número se publicarán trabajos tanto del ámbito de la obstétrica, como de la neonatología, y lo que es más importante, en ellos se enfatiza la relación estrecha entre un cuidado prenatal adecuado con la ocurrencia de resultados neonatales y maternos correctos. En un estudio de casos y controles llevado a cabo en el Hospital General Pediátrico Niños de Acosta Ñu de San Lorenzo en Paraguay, se señala el hallazgo de la asociación entre la presencia de parálisis cerebral y el antecedente de preeclampsia durante su gestación. A partir de estos hallazgos, los cuales no han sido descritos con frecuencia en la literatura médica, los autores proponen la necesidad de tener un mayor conocimiento de cómo las complicaciones obstétricas pueden llevar a desenlaces neonatales adversos; debiéndose llevar a cabo la implementación de intervenciones médicas preventivas tanto en el control prenatal, como en el partoy la atención neonatal.

En un segundo artículo, llevado a cabo en el Centro Materno Infantil Villa María del Triunfo, de Lima, Perenú, los autores evaluaron las complicaciones obstétricas asociados a un control prenatal inadecuado. En êste estudio se encontró que cerca del $40 \%$ de las embarazadas tuvieron un control prenatal deficiente, el-๕ual se asoció con una inadecuada ganancia de peso materna, infecciones del tracto urinario y con anemia.

Finalmente, en una investigación en que se inclüyeron 155 recién nacidos, los autores investigaronelas condiciones de riesgo asociadas al desarrollo de hipotermia neonatal durante su estancia en una unidad de cuidado intensivo; se encontró que cerca del $90 \%$ de los neonatos presentó algún grado de hipotermia y êsta estuvo asociada al nacimiento vía cesárea, preseñcia de restricción en el crecimiento intrauterino y cursarcon anomalías congénitas. Los autores enfatizan que p̧ara evitar este tipo de complicación se requiere una exploración temprana de la existencia de factores de riesggo, con el fin de aplicar un plan de cuidados seguro $\overline{\mathrm{y}}$ de calidad, que sea implementado a tiempo y así disminuir esta complicación neonatal y sus consecuencias.

\section{Correspondencia:}

${ }^{*}$ Ricardo Figueroa-Damián

E-mail: rfd6102@yahoo.com.mx
Fecha de recepción: 07-11-2019

Fecha de aceptación: 11-11-2019 DOI: 10.24875/PER.M19000006
Dr. Ricardo Figueroa Damián EDIFOR Perinatología y Reproducción Humăna

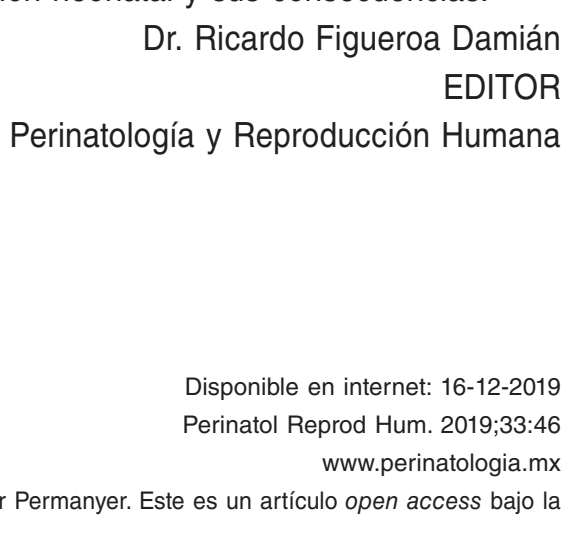

